\title{
DISCUSSION AFTER PAPER BY BOYARCHUK
}

Hesser to Boyarchuk : In your first slide you showed the diagram of Eggen and Sandage relating $[\mathrm{Fe} / \mathrm{H}]$ to age. This diagram still shows a gap between the Population II clusters (47 Tuc) and the disc objects. Dr David Hartwick and I have investigated 3 previously unstudied southern globular clusters all of which appear to have metal abundances comparable to those of other disc objects (Hartwick, F.D.A. and Hesser, J.E.: Astrophys. J. 175, 77, 1972). This leaves us with a possible dilemma, in that the giant branches of 47 Tuc and NGC 6352 do not overlap those of old open clusters like M67 or of field giant stars (Hartwick and Hesser Publ. Astron. Soc. Pacific 84, 813,1972 ), even though the line blanketing, as measured by broadband techniques, is the same for the globular cluster stars as for the disc stars.

Fowler to Krügel: Were you referring to the work of Mezger.

Krugel: Yes, I was asked to do some theoretical calculations on the role of dust.

Fowler: Are you saying that the fact that he observes no helium does not mean that there is none there?

Woolf: For $\mathrm{H}$ II regions near the galactic centre, you cannot resolve the infra-red emission of the $\mathrm{H}$ II regions from that of nearby molecular regions. However, we can separate these in the Orion nebula, and know from this that most of the infra-red emission comes from the molecular region not the $\mathrm{H}$ II region. Therefore in Orion the absorption of UV radiation by internal dust does not substantially affect the ionization of the $\mathrm{H}$ II region. Therefore, the best assumption which we can make for regions near to the galactic centre is that dust is not important there, in which case helium really is deficient.

Audouze: I think that the abundances deduced from the radio recombination lines must critically depend on the temperature of the regions in front of which the measurements are made. Unfortunately this parameter is not always well known.

Krügel: Temperature uncertainties by a factor of two do not affect the results much.

Fowler: Those who are convinced of a 'universal' He abundance should be warned that there are now sophisticated theories of the pre-baryon stage of the Friedmann universe which predict no production of $\mathrm{He}$ in the 'big bang'. $\mathbf{R}$. Wagoner is speaking on these theories at Cracow this week. 